\title{
Simulation of Global Solar Flares and their Effects on Space Weather by using a Laser - Produced Plasma Clouds
}

\author{
A.G. Ponomarenko, Yu. P. Zakharov, V.M. Antonov, E.L. \\ Boyarintsev, A.V. Melekhov, V.G. Posukh and I.F. Shaikhislamov \\ Institute of Laser Physics (ILP), Russian Academy of Sciences, 630090, Novosibirsk, Russia \\ email: zakharov@plasma.nsk.ru
}

Solar flares are of great fundamental and practical interest. In some events the energy release might exceed $10^{26} J$ Tsurutani et al. (2003) and accompanying CME courses extremely severe magnetic storm. Because of very rare occurrence of such global flares a laboratory simulation appears to be an alternative that could provide experimental data necessary for physical understanding of this phenomenon and for verifying theoretical models. Flare involves processes on many widely varying spatial and time scales. In laboratory only some parts of the whole evolution could be attempted for simulation. The present work is focused on the last stage of eruption when a magnetic loop filled with heated plasma is ejected away from the Sun, either itself or pushed by an upcoming jet. The geometry of a flare loop suggests that a magnetic dipole and laser-produced plasma could be used to simulate this structure Nikitin \& Ponomarenko (1995). In the present work similarity criteria that relate laboratory parameters to the natural phenomena, experimental set up and preliminary results are presented.

To relate flare physics to laboratory experiment, three most important similarity criteria have been chosen. A number of previous studies Ponomarenko et al. (2000) show that the energy criteria (relation of characteristic magnetic to the total plasma energy) is the most important one. To eliminate non-MHD effects, the ion Larmor radius should be small enough. The next parameter which we consider to be important is derived from the dimensionless Ampere law. Only when typical current is smaller than characteristic plasma velocity reach and complex magnetic structures like on the Sun could be generated. Analysis yields that only with magnetic moments $>10^{6} \mathrm{G} \cdot \mathrm{cm}^{3}$ and plasma energies $>20 \mathrm{~J}$ all this conditions could be satisfied.

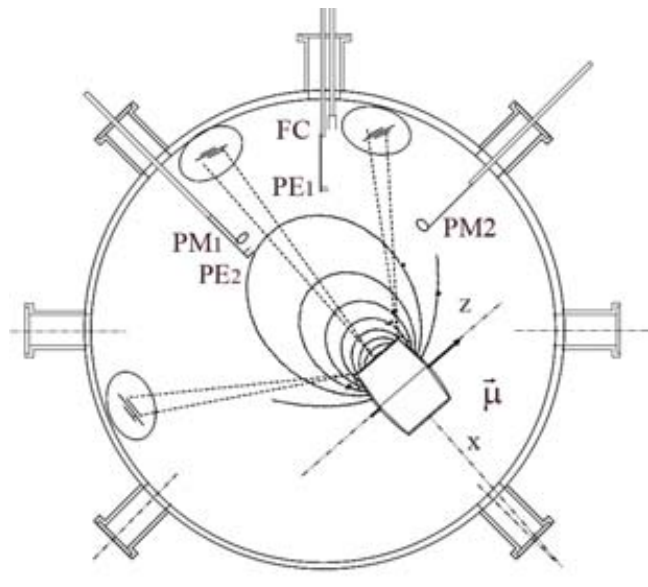

Figure 1.

Specialized KI-1 Facility consists of large $(1 \times 5 \mathrm{~m})$ vacuum chamber and powerful $\mathrm{CO}_{2}$ laser system. First laser beam with energy $\sim 50 \mathrm{~J}$ and pulse duration 100 ns was splitted and focused at two spots on the dipole cover. These side spots separated by the distance of $15 \mathrm{~cm}$ were magnetically tied. After controlled time delay, second laser beam with energy $80 \mathrm{~J}$ was focused between the side spots. Produced plasma consisted mostly of $\mathrm{H}^{+}$, $C^{+4}, C^{+3}$ ions and expanded with velocity $3 \cdot 10^{7} \mathrm{~cm} / \mathrm{s}$. Pulsed coil was used to generate magnetic moment $\mu=10^{7} \mathrm{G} \cdot \mathrm{cm}^{3}$. Magnetic field at the side and central 
spots was 9 and $5 k G$ respectively. Diagnostics consisted of Longmuir and magnetic probes. A fast imaging with time resolution $\sim 10 \mathrm{~ns}$ was used to snap-shot complex spatial structures (figure 1).

Experiments with the plasmas from the side spots showed formation of a quasi-arc structure captured by the field. Probe measurements revealed that at a time $\sim 1 \mu \mathrm{s}$ plasma density at distances $25-40 \mathrm{~cm}$ from the dipole center is about $10^{13} \mathrm{~cm}^{-3}$, which is at least four times bigger than in the case without dipole field. The arc has width $d x \sim 10 \mathrm{~cm}$, depth $d y \sim 10 \mathrm{~cm}$ and moves upward (away from the dipole) with velocity $\sim 10^{7} \mathrm{~cm} / \mathrm{s}$. Photos on figure 2 illustrate the initial (left) and later (right) stages of central piston interaction with a quasi-arc structure produced by injection of two plasmas at the polar regions. Imaging and probe measurements showed that the central exploding plasma transforms in time into expanding and rising loop with $\beta \sim 1$. A most strong effect of its interaction with the arc structure has been observed by means of a remote magnetic probe $\mathrm{PM}_{2}$. When jet interacts with side plasmas its signal increases by at least an order of magnitude up to $\sim 100 \mathrm{G}$. Such field disturbance could not be accounted for by a simple diamagnetism and suggest generation of a new current structure which we associate with the Birkeland current of intensity $\sim 4 k A$ flowing along the loops down to the polar region and back. It is estimated that about $6 \mathrm{~J}$ of energy is stored in the loop current configuration. This energy is supplied by the fast jet when it crosses the arc. Probe measurements confirmed that the jet looses approximately this amount of energy $(\sim 30 \%)$ after crossing the interaction region.
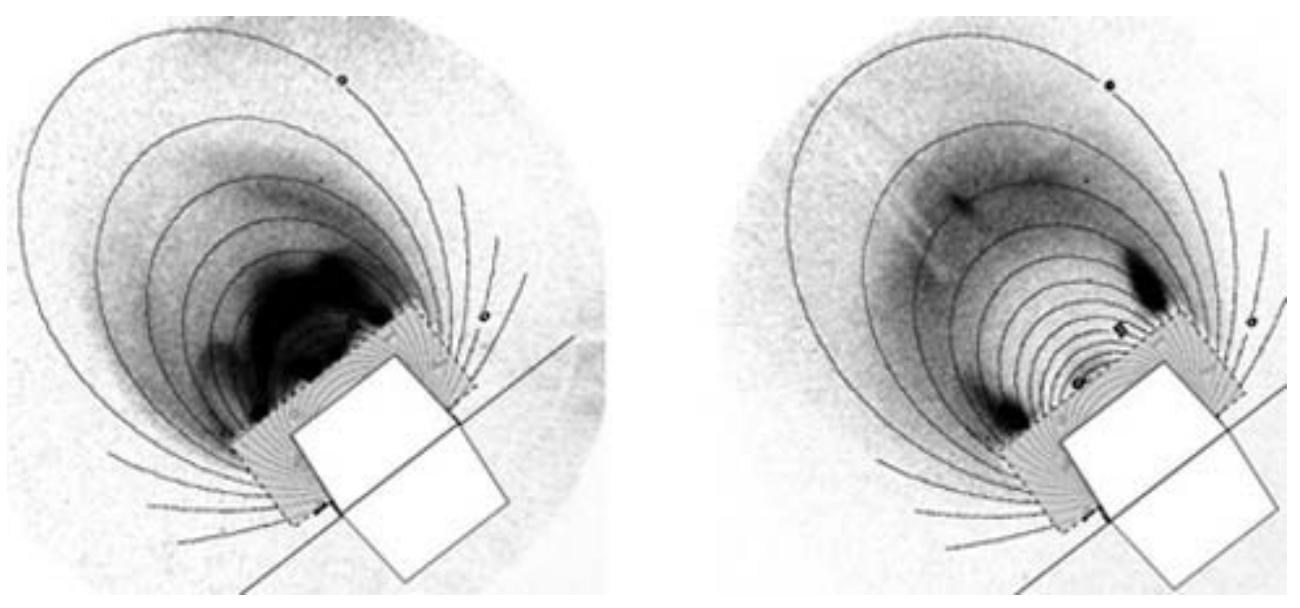

Figure 2.

Preliminary experiments showed that by means of laser-produced plasmas and dipole magnetic field a well localized loop could be generated. Strong magnetic moment and powerful lasers used made it possible to realize parameters close enough to requirements of the most important similarity criteria. Experiments with the up-coming jet interacting with the arc revealed formation of complex current structures. Thus, a possibility to simulate in laboratory high energy explosions in a dipole-like structure with a strong magnetic field on the Sun surface is demonstrated.

This work is supported by SB RAS program.

\section{References}

Tsurutani B.T., Gonzalez W.D., et al. 2003, J. Geophys. Res. 108, No A7, SSH 1-4

Nikitin S.A., Ponomarenko A.G. 1995, Russian J. of Appl. Mech. And Tech. Phys. 36, No 4,3

Ponomarenko A.G et al. 2000, Report ILP-1111 under contract CEA/DAM N 2658/DIR 